INVESTIGACIÓN

Recibido: 09/08/2016 --- Aceptado: 12/12/2016 --- Publicado: 15/06/2017

\title{
EL HUERTO ESCOLAR COMO HERRAMIENTA INNOVADORA QUE CONTRIBUYE AL DESARROLLO COMPETENCIAL DEL ESTUDIANTE UNIVERSITARIO. UNA PROPUESTA EDUCATIVA MULTIDISCIPLINAR
}

The school garden as an innovative tool that contributes to the skills development of the college student. A multidisciplinary educational proposal

Ana María Botella Nicolás1: Universitat de València. España.

ana.maria.botella@uv.es

Amparo Hurtado Soler: Universitat de València. España

amparo.hurtado@uv.es

José Cantó Doménech: Universitat de València. España

jocando@uv.es

El artículo está financiado por el proyecto de innovación educativa L'Hort 2.0 (2014-2015) UVSFPIE_DOCE14-223306 en la modalidad DOCENTIC otorgado por el vicerrectorado de politicas de formación y calidad educativa de la Universitat de València.

\section{RESUMEN}

El huerto escolar es un recurso pedagógico que permite aproximar al alumnado al entorno natural diseñando experiencias interdisciplinares que contribuyan al desarrollo de las competencias básicas. En el ámbito universitario facilita el aprendizaje cooperativo y la adquisición de habilidades socioambientales. El proyecto L'Hort 2.0 pretende crear un entorno virtual basado en el huerto escolar para la administración de contenidos TIC que permitan enlazar las actividades fuera del aula con el contenido de las guías docentes. Se trabaja mediante una metodología activa y colaborativa, en tres fases: preparación y diseño del entorno web en seis áreas temáticas; elaboración de materiales educativos y evaluación de su utilidad en el aula y su eficacia en el proceso de aprendizaje del alumnado. Los resultados obtenidos muestran una elevada implicación del alumnado en el proceso formativo y una mejora en los resultados de evaluación que permite integrar todas las capacidades del alumnado reflejando mejor las competencias alcanzadas.

\footnotetext{
1 Ana María Botella Nicolás: profesora contratada doctora del Departamento de Didáctica de la Expresión musical, plástica y corporal de la Facultad de Magisterio de la Universitat de València. ana.maria.botella@uv.es
} 
Botella Nicolás, A. M., Hurtado Soler, A., Cantó Doménech, J. El huerto escolar como herramienta innovadora que contribuye al desarrollo competencial del estudiante universitario.

\title{
PALABRAS CLAVE
}

Huerto escolar - Multidisciplinariedad - Competencias básicas - Educación superior - Innovación - TIC - Aprendizaje colaborativo

\begin{abstract}
The school garden is an educational resource that allows students to approach the natural environment by designing interdisciplinary experiences that contribute to the development of basic skills. At university level facilitates also a collaborative learning and the acquisition of social and environmental skills. L'Hort 2.0 project aims to create a virtual environment in the school garden based content management for TIC that allows linking the activities outside the classroom with the content of the teaching guides. Working by an active and collaborative methodology in three phases: preparation and design of the web environment in six areas, development of educational materials and evaluation of their usefulness in the classroom and their effectiveness in student learning process. We obtained a high student involvement in the learning process and improved assessment results that can integrate all the capabilities of students to better reflect the skills attained.
\end{abstract}

\section{KEY WORDS}

School garden - Multidisciplinary - Basic skills - Higher education - Innovation ICT - Collaborative learning

\section{A HORTA ESCOLAR COMO FERRAMENTA INOVADORA QUE CONTRIBUI AO DESENVOLVIMENTO DAS COMPETENCIAS DO ESTUDANTE UNIVERSITARIO. UMA PROPOSTA EDUCATIVA MULTIDISCIPLINAR}

\section{RESUMO}

A horta escolar é um recurso pedagógico que permite aproximar o aluno ao entorno natural desenhando experiências interdisciplinares que contribuam ao desenvolvimento das competências básicas. No âmbito universitário facilita o aprendizado cooperativo e a aquisição de habilidades sócio-ambientais. O projeto L'Hort 2.0 pretende criar um entorno virtual baseado em uma horta escolar para a administração de conteúdos TIC que permitam enlaçar as atividades fora da aula com o conteúdo das guias docentes. Trabalha-se mediante uma metodologia ativa e colaborativa, em três fases: preparação e desenho do entorno web em seis áreas temáticas; elaboração de materiais educativos e valoração de sua utilidade em aula e sua eficácia no processo de aprendizagem do aluno. Os resultados obtidos mostram uma elevada implicação do aluno no processo formativo e uma melhora nos resultados de valoração que permite integrar todas as capacidades do aluno refletindo melhor as competências alcançadas. 
Botella Nicolás, A. M., Hurtado Soler, A., Cantó Doménech, J. El huerto escolar como herramienta innovadora que contribuye al desarrollo competencial del estudiante universitario.

\section{PALAVRAS CHAVE}

Horta Escolar - Multidisciplinaridade - Competências básicas - Educação Superior Inovação - TIC - Aprendizagem colaborativa

\section{Cómo citar este artículo}

Botella Nicolás, A. M., Hurtado Soler, A., Cantó Doménech, J. (2017). El huerto escolar como herramienta innovadora que contribuye al desarrollo competencial del estudiante universitario. Una propuesta educativa multidisciplinar. [The school garden as an innovative tool that contributes to the skills development of the college student. A multidisciplinary educational proposal]. Vivat Academia, revista de comunicación, 139, 19-31. doi: http://doi.org/10.15178/va.2017.139.19-31 Recuperado de http:/ / www.vivatacademia.net/index.php/vivat/article/view/935

\section{INTRODUCCIÓN}

Desde el curso 2012-2013 se está llevando a cabo en la Universitat de València una experiencia de innovación educativa (Proyecto L'Hort 2.0) que implica a 26 asignaturas de 6 titulaciones (Grados en Maestro de Primaria, Maestro de Infantil, Pedagogía, Geografía y Medio Ambiente, Filosofía y Lenguas Modernas y sus Literaturas) con la participación de 10 profesores y 1000 alumnos.

El valor del huerto como recurso didáctico depende de la habilidad con que se le maneje y utilice en el proceso de enseñanza aprendizaje, para comprender relaciones de causa y efecto, practicar y aplicar lo que se aprende, utilizarlo como laboratorio en las diferentes asignaturas, aprovechar los recursos del entorno y simultáneamente preparar a los niños y niñas para la vida (Sánchez, Badía \& Hándal, 2009).

Según Ruíz (2003) el concepto de huerto escolar en el sentido educativo actual proviene de los años 70 y es el resultado de la confluencia de tres aspectos: la educación ambiental, los programas de educación para el desarrollo y los proyectos de los movimientos de renovación pedagógica. $Y$ es "la combinación de la concienciación del deterioro del medio ambiente con la educación para la correcta nutrición y la búsqueda de nuevas metodologías" (Ruíz, 2013, p. 25). Actualmente existe un florecimiento del huerto escolar que se hace patente en los numerosos blogs, páginas web y otras publicaciones que lo tratan, pero no tanto desde una perspectiva didáctica.

El trabajo en el huerto escolar facilita el desarrollo de una práctica educativa que implica principalmente la conjunción de tres dimensiones (Ceida, citado en Botella, Hurtado \& Cantó, 2014):

A) Educar en el medio: investigando y trabajando directamente en el medio, relacionando los problemas que afectan a ese entorno cercano con problemáticas más globales.

B) Educar sobre el medio: El huerto es un sistema ecológico, que como tal habrá de ser investigado en su conjunto, teniendo en cuenta los elementos que lo conforman, las interacciones que se dan entre ellos, los cambios que sufre, su organización, y las interdependencias que tiene con respecto a otros sistemas. 
Botella Nicolás, A. M., Hurtado Soler, A., Cantó Doménech, J. El huerto escolar como herramienta innovadora que contribuye al desarrollo competencial del estudiante universitario.

C) Educar a favor del medio: impulsando una serie de valores y actitudes necesarios para un cambio hacia comportamientos más respetuosos con el medio ambiente.

Además, el huerto escolar constituye "un escenario privilegiado para una educación comprometida con la transición hacia modelos sociales más justos y sistemas ambientales capaces de regeneración productiva" (Larrosa, 2013, p. 4).

Con este proyecto educativo se pretende trasladar al aula las actividades desarrolladas en el huerto escolar mediante la creación de un entorno virtual que permitan al docente, por un lado, disponer de recursos didácticos para el desarrollo de sus clases y por otro, establecer una conexión entre el mundo físico y el digital, relacionando las actividades no formales fuera del aula con el contenido de los programas de las guías docentes.

Teniendo en cuenta el marcado carácter tecnológico de los objetos de aprendizaje a elaborar por los alumnos, algunas de las actitudes que promueve para adquirir la competencia tecnológica son:

- Promover actitudes positivas, y al mismo tiempo críticas, hacia el uso de las tecnologías de la información y la comunicación.

- Potenciar la colaboración en las acciones educativas, tanto entre el profesorado como entre el alumnado y promover la autonomía en los procesos de enseñanzaaprendizaje entre el alumnado.

- Utilizar las tecnologías como instrumentos potenciadores de la creatividad para generar recursos educativos.

- Reflexionar sobre las prácticas de aula para innovar y mejorar la labor docente. Adquirir hábitos y destrezas para el aprendizaje autónomo y cooperativo y promoverlo entre los estudiantes.

- Conocer y aplicar en las aulas las tecnologías de la información y de la comunicación.

- Discernir selectivamente la información audiovisual que contribuya a los aprendizajes, a la formación cívica y a la riqueza cultural.

- Comprender la función, las posibilidades y los límites de la educación en la sociedad actual y las competencias fundamentales que afectan a los colegios de educación primaria y a sus profesionales.

- Conocer modelos de mejora de calidad.

Por otra parte, los alumnos adquieren otras competencias relacionadas con los contenidos teóricos de las asignaturas en las que se desarrollan las actividades y se utilizan los recursos TIC destacando la competencia matemática, la interacción con el medio físico, natural, social y cultural, la competencia lingüística y la competencia artística.

\section{OBJETIVOS}

El objetivo principal del proyecto, es la adquisición y refuerzo de las competencias generales del grado sobre todo relacionadas con la transferencia del conocimiento y el ámbito profesional. Para su consecución se han establecido unas directrices 
Botella Nicolás, A. M., Hurtado Soler, A., Cantó Doménech, J. El huerto escolar como herramienta innovadora que contribuye al desarrollo competencial del estudiante universitario.

orientadas a: Promover el trabajo cooperativo y colaborativo de los alumnos y entre las escuelas y la universidad a través del Prácticum; Impulsar la calidad docente mediante la creación de materiales educativos TIC y una reflexión profunda sobre los criterios de evaluación y la metodología de trabajo en el aula; Generar nuevos recursos con enfoque interdisciplinar y transversal que permitan abordar con eficacia situaciones de aprendizaje en contextos diferentes aplicando las nuevas tecnologías en situaciones cotidianas.

Además, potencia las competencias transversales mediante el aprendizaje cooperativo y la adquisición de habilidades sociales de integración, igualdad y solidaridad, siendo un excelente recurso didáctico para las áreas de las Ciencias Experimentales y Sociales (física, química, biología, matemáticas, geografía, medioambiente, tecnología agraria) y las áreas de Lingüística y Expresión Plástica y Musical.

Así, el desarrollo competencial a través de este proyecto rompe con la parcelación y el academicismo estimulando un aprendizaje constructivo, creativo y de producción de conocimientos, facilitando el desarrollo integral educativo, tal y como opinan Kincheloe, Steinberg \& Villaverde (2004). Según Margalef (2005) el profesorado brinda al alumnado la oportunidad de relacionar la teoría y la práctica, de realizar aprendizajes procedimentales y de reconstruir sus aprendizajes previos y marcos referenciales a partir de la reflexión.

En este contexto, se pueden trabajar tanto los contenidos como los procedimientos y actitudes siendo un marco idóneo para tratar otros temas transversales (sostenibilidad, hábitos saludables, valores y ciudadanía).

Para Escanilla (2008) el enfoque competencial se sustenta en cuatro bases: sociológica, psicológica, epistemológica y pedagógica que nos muestran el porqué debemos trabajar las competencias, qué las identifica y cómo desarrollarlas a través de proyectos. Si tomamos la definición de competencia de Lasnier (2000), una competencia es un saber hacer complejo, resultado de la integración, movilización y adecuación de capacidades, habilidades (pueden ser de orden cognitivo, afectivo, psicomotor o social) y de conocimientos (conocimientos declarativos) utilizados eficazmente en situaciones que tengan un carácter común (situaciones similares, no generalizable a cualquier situación). Atendiendo a esta definición el proyecto implica tanto el uso de habilidades cognitivas, como afectivas y de relaciones, pero su potencialidad como recurso didáctico dependerá de la habilidad con que se maneje y se utilice en el proceso de enseñanza aprendizaje. Así, se contribuye a que alumnado y profesorado desarrollen capacidades de aprendizaje que estén fundamentadas sobre estructuras de conocimiento sólidas, y por otra, ofrece un amplio abanico de aptitudes que incluyen, entre otras muchas, la capacidad para el esfuerzo y la superación personal, la obligación de interpretar y adaptar información, trabajar en equipo, potenciar la iniciativa personal, resolver situaciones inesperadas, convivir en grupo y resolver los conflictos de manera adecuada (Cantó, Hurtado \& Gavidia, 2013).

Este protagonismo del alumnado hacia su propio proceso de aprendizaje requiere de una nueva estrategia de evaluación educativa vista como una evaluación personalizada y centrada en el alumno y en sus capacidades. Además la evaluación debe mejorarse orientándose hacia un proceso formativo con un alumnado activo y 
Botella Nicolás, A. M., Hurtado Soler, A., Cantó Doménech, J. El huerto escolar como herramienta innovadora que contribuye al desarrollo competencial del estudiante universitario.

responsable en sus aprendizajes, según Bowden y Marton (2012), y un profesorado con un papel interactivo y secuencial, para Bembenutty (2009). Un correcto uso del feedback en la evaluación formativa mejora el aprendizaje y el resultado de los estudiantes (Aranda, 2013).

\section{METODOLOGÍA}

Siguiendo a Taylor y Bogan (2000), la metodología empleada ha sido la cualitativa como método que produce datos descriptivos. El diseño de las actividades experimentales en el huerto escolar desde el aprendizaje por descubrimiento, invita a los estudiantes a utilizar las ideas y conocimientos previos que poseen sobre las tareas que deben desarrollar en cada actividad (Vera, 2015). El registro de los procesos por competencias se realiza mediante el estudio de casos, ya que permite realizar el seguimiento a los estudiantes durante las diferentes fases de aplicación. En palabras de Paula (2004, p. 243), el diseño del estudio de casos "se inscribe dentro de la lógica que guía las sucesivas etapas de recogida, análisis e interpretación de datos de los modelos cualitativos, con la peculiaridad de que el propósito de la investigación es el estudio intensivo y profundo de uno o pocos casos de un fenómeno".

Se trata de una propuesta educativa integradora que fomenta el aprendizaje autónomo y comprometido del alumno a través de una metodología que se sintetiza en tres aspectos según Botella, Hurtado, Martínez \& Talavera (2014):

- Actividad investigadora: se pretende que el alumnado plantee dudas, formule hipótesis, diseñe experimentos, conectando sus conocimientos con nuevas fuentes de información para obtener sus propias conclusiones.

- Trabajo colaborativo: el alumno/a organiza su propio trabajo y el de grupo mediante el reparto de tareas, el intercambio de la información y el compromiso con el resto de compañeros y compañeras.

- Globalidad: las disciplinas interaccionan entre sí estableciendo flujos de intercambio donde todo está relacionado.

Mediante una metodología activa y colaborativa, los estudiantes han tenido que preparar y estructurar en un entorno web, sus actividades, abarcando en total seis áreas temáticas: ciencias experimentales y salud, medioambiente y territorio, matemáticas, lengua y literatura, plástica y música y educación en valores y ciudadanía. En dicho entorno (www.uv.es/hort), se han alojado los distintos objetos de aprendizaje TIC diseñados y relacionados con los contenidos de las asignaturas de grado.

El diseño de los objetos de aprendizaje utilizados en el aula se ha realizado utilizando software libre. Entre los objetos desarrollados hay que destacar: actividades y materiales docentes con juegos interactivos (Jclik, Hotpotatoes), blog, wiki, webquest...; realización de vídeos de prácticas de laboratorio (volumetría, fluidos, rocas, ecosistemas...); edición de archivos de audio para el desarrollo de competencias lingüísticas y musicales; elaboración de mapas conceptuales con CmapTools; diseño de materiales para PDI; elaboración de cartografía temática y 
Botella Nicolás, A. M., Hurtado Soler, A., Cantó Doménech, J. El huerto escolar como herramienta innovadora que contribuye al desarrollo competencial del estudiante universitario.

fotointerpretación; etc. A modo de ejemplo, algunos de los materiales realizados por el alumnado han sido, para cada área, los siguientes:

- TIC: PDI, manejo de programas audiovisuales y sistemas de autor y trabajo cooperativo en web 2.0.

- Ciencias y Matemáticas: propuestas didácticas (guías, juegos interactivos, actividades), contenidos teóricos (mapas conceptuales, juegos, vídeos y webquest).

- Medioambiente y Sociales: procesos naturales (vídeos), escala (cartografía digital), patrimonio y cultural (videos y juegos), actitudes medioambientales responsables (guías de buenas prácticas), gestión de recursos (hojas de datos)

- Plástica y Música: formas, colores, materiales, sonidos, estructura musical (partituras interactivas, vídeos y archivos de audio con temas del huerto)

- Lengua y Literatura: gramática y vocabulario (vídeos y audio de fonética, juegos inter y mat multimedia), literatura (lecturas, audio poemas, itinerarios literarios)

- Valores y Ética: atención a la diversidad (juegos de rol, etc.)

\section{DISCUSIÓN}

Entre los resultados obtenidos en el proyecto L'Hort 2.0 destacamos la implementación de dos nuevas asignaturas que se han desarrollado en la Facultad de Magisterio de la Universitat de València en el grado de Maestro de Primaria durante los cursos 2012-2013, 2013-2014 y 2014-2015.

\subsection{Diseño de materiales educativos ( $3^{\circ}$ curso $)$ :}

La asignatura se dirige a cuatro grupos de alumnos del itinerario TIC (145 alumnos). El objetivo es la adquisición de destrezas tecnológicas en el uso de aplicaciones multimedia (vídeo, audio...), web 2.0 (blog, wiki, webquest...) y sistemas de autor (Jclik, Hotpotatoes...) para el diseño de materiales educativos.

La estructura de la materia se organiza en seis bloques: gestión de materiales educativos en red, PDI, aprendizaje cooperativo (wiki, webquest), entornos de comunicación en el aula (blog, redes), sistemas de autor, entornos virtuales (Moodle). En cada bloque los alumnos realizaron una actividad relacionada con los contenidos impartidos bien en grupo como individualmente. Las ejemplificaciones y los tutoriales de los programas se realizaron utilizando los materiales diseñados en el proyecto L'Hort 2.0. Estos materiales didácticos fueron evaluados por los alumnos mediante la implementación de un diario de incidencias y un informe final de la asignatura en el que emitieron su opinión crítica acerca del proceso, los materiales y las dificultades observadas.

Para la elaboración de las actividades con los sistemas de autor se realizó una salida al huerto donde tomaron material gráfico (vídeos y fotografías) que utilizaron en el diseño. Por otra parte, realizaron una reflexión crítica acerca de las potencialidades del huerto escolar como recurso pedagógico para el desarrollo de las competencias básicas de la etapa obligatoria.

La evaluación se llevó a cabo teniendo en cuenta de forma ponderada todas las actividades realizadas tanto dentro como fuera del aula, así como la participación y el 
Botella Nicolás, A. M., Hurtado Soler, A., Cantó Doménech, J. El huerto escolar como herramienta innovadora que contribuye al desarrollo competencial del estudiante universitario.

interés en la asignatura a través de la observación, el diario de incidencias y el informe final.

\subsection{TIC como recurso didáctico en ciencias y matemáticas ( $4^{\circ}$ curso):}

La asignatura se dirige a tres grupos (95 alumnos) con dos perfiles distintos: estudiantes del itinerario de Ciencias (28 alumnos) que ya habían diseñado propuestas multidisciplinares pero que carecían de conocimientos sobre herramientas TIC y estudiantes del itinerario TIC (67 alumnos) y desconocían cómo plantear una propuesta didáctica con contenidos de ciencias y de matemáticas. Las primeras sesiones sirvieron para homogeneizar los grupos introduciendo los conocimientos de que carecían y poder partir de un punto común en el resto de sesiones.

En las siguientes sesiones los alumnos distribuidos en grupos de 3 diseñaron una propuesta didáctica sobre soporte informático (blog). El blog debía enmarcarse en un contexto a elegir por cada grupo y debía contener al menos tres bloques en los que seleccionaron contenidos de ciencias y matemáticas del curriculum de un nivel concreto de primaria también seleccionado por ellos. Además el blog debía contener una justificación de la propuesta, herramientas y proceso de evaluación, posibles trasversalidades y una guía para el profesor, con el fin de que estas propuestas pudieran ser compartidas con otros profesionales docentes. Dado el carácter tecnológico de la asignatura se solicitó que los alumnos diseñaran sus propios materiales educativos utilizando software libre y sistemas de autor (jclik, Hotpotatoes, Geogebra...) y realizasen una búsqueda exhaustiva y crítica sobre los recursos digitales educativos existentes en la web.

Las propuestas didácticas se desarrollaron en gran medida durante las horas de clase para facilitar el feedback con el profesor que mostró materiales diseñados en el proyecto L'Hort 2.0 como ejemplificación de algunos de los contenidos (utilización de software y herramientas de internet, un modelo de propuesta didáctica, selección de contenidos en la web...).

Dentro de las características de la propuesta, ésta debía contemplar contenidos en ciencias y matemáticas a desarrollar en el huerto escolar. Para facilitar la elaboración de las actividades se realizaron dos visitas al huerto. En la primera con el objetivo de recopilar información (digital y escrita): tamaño, distribución, cultivos... con la que diseñar las actividades y la segunda con el fin de llevar a la práctica una de las actividades diseñadas y así comprobar la viabilidad de la misma. En esta segunda salida cada grupo realizó una evaluación de las actividades propuestas por sus compañeros. De esta forma los alumnos formaron parte del proceso evaluativo de la asignatura.

Durante el desarrollo de la asignatura los grupos expusieron el proceso de trabajo y se realizó una evaluación de las exposiciones en las que participaron todos los alumnos aportando ideas y mejoras en los trabajos de los compañeros. Al finalizar la asignatura cada grupo realizó una exposición final a modo de comunicación y con un tiempo reducido (10 minutos) con el fin de observar y evaluar su capacidad de síntesis, la presentación y la justificación de la propuesta. 
Botella Nicolás, A. M., Hurtado Soler, A., Cantó Doménech, J. El huerto escolar como herramienta innovadora que contribuye al desarrollo competencial del estudiante universitario.

En la evaluación del alumnado se tuvieron en cuenta todas las fases de aprendizaje valorándose de forma individual y grupal: la participación, un control de contenidos correspondiente a las primeras sesiones, las tareas realizadas en clase, la actividad del huerto y la evaluación de los compañeros, exposición de la propuesta y el resultado final del blog.

Además también cabe destacar las distintas propuestas realizadas por el alumnado participante en el marco de las asignaturas que forman parte del Proyecto. Para poder llevar a cabo su presentación y compartir así la experiencia, se organizó a finales de junio de 2015, la “I Jornada de estudiantes L'Hort 2.0: propostes educatives multidisciplinàries". En ella se presentaron los resultados y se analizaron distintas implementaciones prácticas multidisciplinares realizadas por los alumnos en las siguientes áreas:

- Área de temática de Ciencias y Matemáticas:

- Elaboración una Wiki en una propuesta de ciencias y matemáticas contextualizada: la Hortipedia.

- Diseño de una actividad multidisciplinar centrada en una visita al Bioparc de Valencia

- Propuesta de ciencias y matemáticas contextualizada en un entorno rural: Molí del Pas

- Una propuesta de ciencias multidisciplinar en el contexto del huerto escolar.

- Blog contextualizado en la estación espacial como soporte de una propuesta de ciencias y matemáticas

- Área de temática de Ciencias Sociales y Educación en Valores:

- Paraula de un llauraor

- L'incontro con Vincent, una microstoria che racconta una intera comunità

- El cor de l'horta de la costera

- Integridad y cooperación ¿los olvidados del siglo XXI?

- El blog como recurso en la transmisión de experiencias sobre el biohuerto escolar

- El éxodo. Una generación en busca de mejores tierras donde labrar un futuro

- Área de temática de Educación Artística:

- ¿Qué esconde la música de Haydn?

- La audición, puerta al conocimiento

- Què ha vist l'arbre? El pas del temps en la comarca de l'Horta de València

- Área de temática de Lengua y literatura:

- Aprofitament didàctic de l'entorn escolar: La Marjal Pego-Oliva

- La Rondalla va per l'Horta

- L'hort 2.0 i la literatura valenciana: Ruta Carles Salvador

- A riu regirat: Itinerari per l'antic camí del Pouet de Campanar 
Botella Nicolás, A. M., Hurtado Soler, A., Cantó Doménech, J. El huerto escolar como herramienta innovadora que contribuye al desarrollo competencial del estudiante universitario.

- Ruta historicoliterària per Silla i l'Albufera

Con ello, se consiguió cubrir gran parte de los objetivos planteados en el Proyecto, además de ofrecer un marco de divulgación y valoración de las propuestas didácticas elaboradas por los alumnos en materia de innovación educativa, fomentando a la vez, la colaboración e implicación del alumnado en el proceso de aprendizaje y el desarrollo de las asignaturas de los grados, así como su participación en programas de innovación educativa.

\section{CONCLUSIÓN}

Sin lugar a dudas, la experiencia que ha supuesto la implementación y el desarrollo del proyecto L'Hort 2.0, ha sido todo un éxito ya que ha permitido establecer una conexión entre el mundo físico, social y digital, enlazando las actividades no formales fuera del aula (itinerarios y visitas al huerto escolar) con el contenido de los programas de las guías docentes de las asignaturas de grado diseñando actividades de exploración, introducción de conceptos, estructuración, modelización y aplicación en un entorno virtual basado en el huerto escolar.

El uso de las tecnologías para el diseño de materiales TIC es un instrumento potenciador de la creatividad y permite mejorar la labor docente promoviendo el aprendizaje autónomo y cooperativo de los alumnos. Sin embargo, aunque los alumnos se adaptan fácilmente al uso de herramientas TIC, encuentran bastantes dificultades a la hora de preparar materiales multidisciplinares. Además a menudo proponen recursos difíciles de implementar en aulas de primaria y pierden de vista la temporalización, así como la evaluación de las propuestas.

En cuanto a la evaluación de los recursos educativos utilizados por el profesor, la mayor parte de los alumnos los han percibido como adecuados aunque han manifestado algunas dificultades a la hora de seguir los tutoriales cuando utilizan los ordenadores de la Facultad. Ello es debido a la configuración de los equipos de las aulas de informática de la Facultad que no permite conservar configuraciones personales ni actualizar el software utilizado. La mayor parte de las incidencias se deben a dificultades técnicas en la utilización de los programas.

Además, el gran número de propuestas y el hecho de tenerlas un lugar determinado donde se han alojado y compartido, ha sido muy bien recibido por parte del alumnado, ya que suponía una puesta en valor del trabajo realizado. A través de los informes finales los alumnos han emitido algunas valoraciones y sugerencias sobre la metodología de las asignaturas mostrando su descontento por la sobrecarga de trabajo y la dificultad de las actividades y valorando muy positivamente el fomento del trabajo autónomo y el incremento de destrezas y habilidades. En cuanto a la evaluación formativa muestran satisfacción en la diversidad de herramientas utilizadas por el profesor y valoran muy positivamente los trabajos realizados en grupo y las actividades fuera del aula.

Pensamos que la propuesta ha fomentado el aprendizaje cooperativo de los estudiantes y su implicación en el desarrollo de los materiales docentes como parte fundamental del proceso de aprendizaje y como elemento de desarrollo competencial, como, por ejemplo, la participación en el diseño de itinerarios, visitas 
Botella Nicolás, A. M., Hurtado Soler, A., Cantó Doménech, J. El huerto escolar como herramienta innovadora que contribuye al desarrollo competencial del estudiante universitario.

al huerto y elaboración de propuestas didácticas y proyectos TIC, la realización de tutorías virtuales, foros y blog temáticos o la evaluación interactiva entre profesoralumno mediante cuestionarios de calidad sobre los materiales didácticos elaborados por el profesor (idoneidad, aplicabilidad, accesibilidad...). De este modo, los estudiantes han formado parte del proceso de selección de actividades y de su organización e imbricación dentro de los materiales pedagógicos elaborados.

Por otra parte, el aprendizaje colaborativo ha ayudado a practicar estrategias diferenciadas de la clase magistral puramente expositiva. Así, la asignatura no sólo se ha convertido en el lugar de trasvase de conocimiento sino también en el lugar ideal donde intercambiar experiencias relacionadas con la docencia. Además la exposición de trabajos, parte fundamental del proceso de trabajo colaborativo, ha devenido en un hecho único del que todas las partes implicadas, creadores, docentes y compañeros de aula salen beneficiados. Se ha pretendido con ello convertir el aula qué mejor lugar para ello- en un foro de debate centrado en la educación, en la renovación pedagógica y, en la medida de lo posible, en un lugar de investigación centrado en el proceso de enseñanza-aprendizaje y donde el saber se genera de forma cooperativa.

Finalmente, el proyecto de innovación educativa L'Hort 2.0 ha permitido al profesorado implicado disponer de un recurso didáctico que fomenta la implicación del alumnado y su responsabilidad sobre el proceso de aprendizaje, facilitando así la aplicación de una metodología de evaluación formativa que tenga en cuenta todos los aspectos relacionados con el aprendizaje.

\section{REFERENCIAS}

Aranda, A. F., López-Pastor, V., Castejón, J. \& Romero, R. (2013). La evaluación formativa en docencia universitaria y el rendimiento académico del alumnado. Aula Abierta, 41(2), 23-34.

Bembenutty, H. (2009). Teaching effectiveness, course evaluation, and academic performance: The role of academic delay of gratification. Journal of Advanced Academics, 20, 326-355.

Botella, A. M., Hurtado, A. \& Cantó, J. (2014). Las competencias básicas a través del huerto escolar: una propuesta de proyecto de innovación, en J. J. Maquilón Sánchez, \& N. Orcajada Sánchez (Eds.). Investigación e innovación en formación del profesorado (pp. 173-182). Murcia: Servicio publicaciones Universidad de Murcia (Edit.um).

Botella, A. M., Hurtado, A., Martínez, S. \& Talavera, M. (2014). Interpretando el paisajes a través de los sentidos. Una experiencia para la formación del profesorado, en $2^{\circ}$ congreso Universitat de València -Institutos de Estudios Comarcales "Medio ambiente y desarrollo territorial" (pp. 243-252). 
Botella Nicolás, A. M., Hurtado Soler, A., Cantó Doménech, J. El huerto escolar como herramienta innovadora que contribuye al desarrollo competencial del estudiante universitario.

Bowden, J. \& Marton, F. (2012). La Universidad. Un espacio para el aprendizaje. Madrid: Narcea.

Cantó, J., Hurtado, A. \& Gavidia, V. (2013). El huerto escolar: un enfoque trasversal y multidisciplinar para desarrollar competencias, en Jornadas sobre investigación y didáctica en ESO y Bachillerato. Madrid: Santillana.

Ceida. (1999). Huerto Escolar. País Vasco: Departamento de ordenación del territorio, vivienda y medio ambiente.

Escamilla, A. (2008). Las competencias básicas. Claves y propuestas para su desarrollo en los centros. Barcelona: Graó.

Kincheloe, J., Steinberg, S. \& Villaverde, L. (2004). Repensar la inteligencia: Hacer frente a los supuestos psicológicos sobre enseñanza y aprendizaje. Madrid: Morata.

Larrosa, F. J. (2013). Huertos escolares de la región de Murcia. (Trabajo inédito de fin de licenciatura). Universidad de Murcia, España.

Lasnier, F. (2000). Reússir la formation par compétences. Montreal: Guérin.

Margalef, L. (2005). Innovar desde dentro: transformar la enseñanza más allá de la convergencia europea. Revista Iberoamericana de Educación, 37(3), 1-13.

Paula, Isabel (2004). Cuando el síndrome del calendario amenaza: el estrés laboral en educación, en Premios nacionales de investigación educativa (pp. 235-260). Madrid: Ministerio de Educación y Ciencia CIDE.

Ruíz, M. E. (2013). El uso del huerto urbano como recurso didáctico para la enseñanza de matemáticas a los alumnos de $2^{a}$ curso de diversificación. (Trabajo inédito de fin de máster). Universidad de Valladolid, España.

Sánchez, S., Badía, E. \& Hándal, E. (2009). El huerto como recurso escolar. San Salvador: MINED.

Taylor, S. \& Bogdan, R. (2000). Introducción a los métodos cualitativos. México: Ediciones Paidós.

Vera, J. A. (2015). La huerta escolar como estrategia didáctica para el desarrollo de competencias científicas en la institución educativa maestro Pedro nel Gómez. (Tesis inédita de maestría). Universidad Nacional de Colombia (sede Medellín), Colombia. 
Botella Nicolás, A. M., Hurtado Soler, A., Cantó Doménech, J. El huerto escolar como herramienta innovadora que contribuye al desarrollo competencial del estudiante universitario.

\section{AUTORES:}

\section{Ana María Botella Nicolás:}

Doctora en pedagogía por la Universitat de València. Es Licenciada en Geografía e Historia, especialidad Musicología y maestra en Educación Musical, por la Universidad de Oviedo. Grado profesional en la especialidad de piano. Durante el año 2001 obtiene por oposición plaza en el cuerpo de profesores de música de enseñanza secundaria en Alicante (actualmente en excedencia). Ha presentado diversas comunicaciones en jornadas y congresos sobre didáctica de la música así como distintas publicaciones. Es profesora contratada doctora del departamento de didáctica de la Expresión Musical, Plástica y Corporal de la Facultad de Magisterio de la Universitat de València. Forma parte de la Comisión de Coordinación Académica del Master Universitario en Profesor/a de enseñanza secundaria de la UVEG y del Máster de Investigación en didácticas específicas. Desde Febrero de 2015 dirige el aula de música.

http://orcid.org/0000-0001-5324-7152

\section{Amparo Hurtado Soler:}

Doctora en Biología. Profesora del Dpto. de Didáctica de las Ciencias Experimentales y sociales. Imparte docencia en las asignaturas de los itinerarios de Tecnologías de la información y comunicación (TIC) y Ciencias y matemáticas del grado de maestro de primaria, investigación básica en didáctica de las ciencias en el máster de didácticas específicas y tutorización del prácticum de magisterio. Dirección de TFM y TFG centrado en el uso de las TIC en educación y en la innovación educativa. Coordinadora del proyecto de innovación educativa L'Hort 2.0 integrado por 10 profesores de 6 áreas de conocimiento y 2 universidades. Líneas de investigación relacionadas con la salud y medioambiente, la innovación educativa y las TIC.

http://orcid.org/0000-0001-6627-2269

\section{José Cantó Doménech:}

Es licenciado en Ciencias Físicas y Doctor en Física. Actualmente, Ayudante Doctor del Departamento de Didáctica de las Ciencias Experimentales y Sociales de la Universitat de València. Su experiencia docente se reparte entre el Grado de Maestro/a en Educación Infantil y Primaria y el Máster de Investigación de Didácticas Específicas. Su investigación se centra en la formación del profesorado y en la enseñanza de las ciencias en la etapa de infantil y primaria. En el marco de dicha investigación ha realizado distintas estancias en la Universidad de Módena y Reggio-Emilia (Italia) y ha participado en proyectos europeos como Fibonacci. Participa como investigador en proyectos financiados con fondos públicos, ha publicado artículos en revistas como Uni-Pluri/versidad, Didáctica de las Ciencias Experimentales y Sociales, Alambique... y es revisor de distintas revistas científicas de reconocido prestigio en su ámbito.

https://scholar.google.es/citations?user=eUpH-SYAAAAJ\&hl=es 